\title{
Cocaine contaminant Levamisole-induced polyangiitis and necrosis of the nasal cavity - a Scottish case series*
}

Douglas Graham Andrew', Robert Adrian Scott' ${ }^{1}$ Agata T Kochman², Natarajan Balaji ${ }^{1}$

'ENT, Monklands Hospital, Airdrie, United Kingdom

2 Pathology, Monklands Hospital, Airdrie, United Kingdom
Rhinology Online, Vol 2: 21 - 24, 2018

http://doi.org/10.4193/RHINOL/18.069

${ }^{*}$ Received for publication:

August 31, 2018

Accepted: December 9, 2018

Published: February 9, 2019

\begin{abstract}
Background: Common cocaine cutting agent Levamisole is known to cause agranulocytosis. However, a lesser known public health issue is levamisole-induced granulomatosis with polyangiitis. This case series explores this link.
\end{abstract}

Methods and Results: A three case-series report with findings confirmed through clinical history, examination, biopsy and urine toxicology screens.

Conclusions: Our case-series highlights a possible link between levamisole and extensive necrosis of the nasal cavity; caused by polyangiitis with granuloma formation and secondary vasculitis. A high degree of suspicion is needed if young patients present with ANCA positive vasculitis, or in patients with cocaine use, if there is a disproportionate destruction of tissues- particularly the lateral wall of the nose. If diagnosed and treated early, this can be lifesaving.

Key words: cocaine, levamisole, nasal necrosis, polyangiitis, granulomatosis

\section{Introduction}

The use of the veterinary anti-helminthic agent Levamisole as a cutting agent for cocaine has been well documented ${ }^{(1)}$. There have also been links as a causative agent for secondary vasculitic lesions mimicking antineutrophil cytoplasm antibodies (ANCA)positive vasculitis including polyangiitis with granulomatosis (Wegener's granulomatosis) and agranulocytosis with known systemic and localised effects. The commonest effect are cutaneous manifestations, with haemorrhagic bullae or necrosis ${ }^{(2-4)}$. However, difficulty with detection, due to a short half-life and variable concentrations as a cutting agent, has meant a direct link between these systemic and localised conditions are poorly documented or supported ${ }^{(5)}$.

Our case series of three young patients, will help support evidence of the causative link between confirmed levamisole and extensive necrosis of the nasal cavity seen in these patients. These were confirmed with clinical history, examination, biopsy and urine toxicology screens.

\section{Case reports}

\section{Case 1}

This 27-year-old lady was referred by her GP due to sinus pain, nasal congestion, crusting and intermittent epistaxis. Nasendoscopy showed complete destruction predominantly of the midnasal septum, but also extending posteriorly and into the lateral walls (Figure 1 and 2). She underwent debridement and biopsy of her right and left nasal floor and lateral walls.

Nasal biopsy (Figure 3) demonstrated chronic inflammatory changes, with no granulomas. There was evidence of fibrinoid necrosis and minimal intimal inflammation in the arteries, segmental fibrinoid necrosis of the vein wall as well as perivascular chronic inflammatory cell infiltrate. She admitted to unquantifiable, recreational cocaine use. Urine samples tested positive for cocaine and Levamisole.

Immunologically, she was persistently strongly ANCA positive; MPO titres negative; PR3 positive with variable titres (11.0, 6.2 $\mathrm{IU} / \mathrm{mL}$ ); RF negative; CRP and ESR were within normal limits and her eosinophil count was 0.5 . 


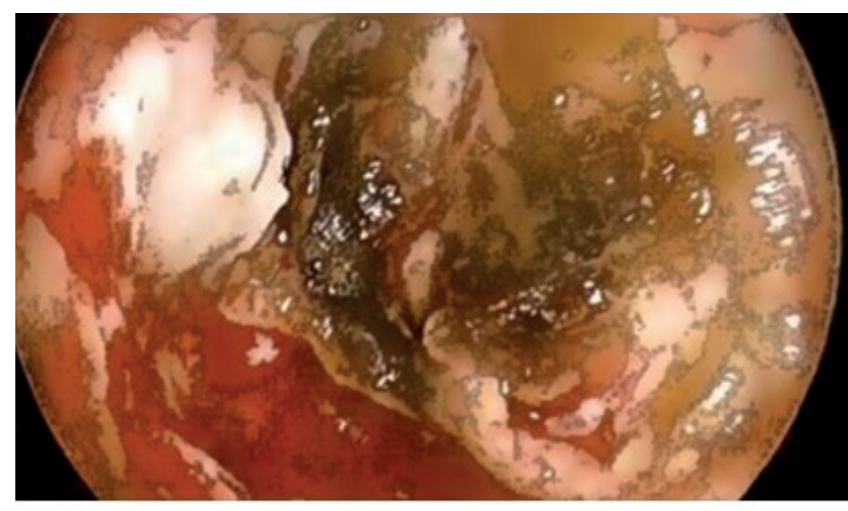

Figure 1. FNE showing extensive ulceration and necrosis of later wall, floor and septum

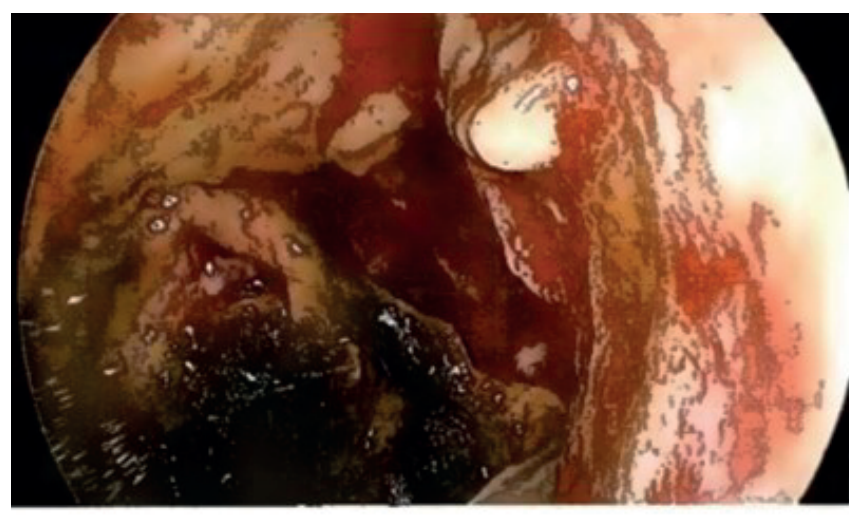

Figure 2. FNE showing further ulceration and necrosis.
Rheumatology diagnosed her as a probable localised granulomatosis with polyangiitis; the diagnostic yield from nasal biopsies in GPA is low ${ }^{(6)}$ and her presentation had gone beyond granuloma, with evidence of bony erosion on biopsy and nasendoscopy. She was asymptomatic of skin, ocular, neurological, upper respiratory or GI manifestations for vasculitis. She was treated with a two weeks course of Prednisolone and subsequently Methotrexate, as well as nasal douching, naseptin and bactroban following debridement.

In May 2016, her isolated vasculitic manifestations had improved dramatically and she had normal mucosa, except the large septal perforation. She denied any further cocaine use.

\section{Case 2}

This 25-year-old gentleman was referred by his GP due to burning pain, a blocked sensation, nasal crusting and bleeding from the nose; thought initially to be from accidental inhalation of chassis acid. On further questioning, he admitted to weekly cocaine use. His urine sample tested positive for cocaine, as well as levamisole. Nasendoscopy revealed extensive crusting and necrosis on the right side of the septum; also involving the right lateral wall and the left side of the septum. He underwent debridement and biopsy of the affected areas.

Biopsy, shown in Figure 4, revealed reactive changes, including fibrotic lamina propria and chronic inflammation. There were metaplastic changes in the surface of the epithelium and within some acini. There were also almost circumferential fibrin deposits in the walls of the small arteries, which appeared limited to the inner layer of the vessel wall, minimal intima inflammation and perivascular chronic inflammation, segmental vein fibrinoid necrosis, patchy thrombosis of capillary vessels but no granulomas. This appearance was deemed inconsistent with granulomatosis with polyangiitis, and more consistent with vasculitis secondary to ongoing inflammation or due to levamisole contamination.

Immunologically, his ANCA Titre tested positive (1/40 p-ANCA),
MPO, PR3, ANA, ESR were negative. His eosinophils were 0.1. He complained of no systemic symptoms and, following debridement, his symptoms dramatically improved and did not receive any further treatment.

\section{Case 3}

This 21-year-old underwent bilateral anterior ethmoidal artery ligation following a traumatic nasal bone fractures. At the time, he was noted to have a large septal perforation with exposed cartilage. Biopsies (Figure 5) were taken and revealed further fibrinoid necrosis and inflammation of the arteries and veins, as well as non-specific, ulcerated fragments of cartilage and a separate fragment of granulation tissue. Urine tested positive for cocaine and levamisole.

Immunologically, he had a low titre ANA (1/80 homogenous). ANCA, ESR were negative and his eosinophils were 0.1. He underwent further debridement and septorhinoplasty, but his septal perforation was managed conservatively.

\section{Discussion}

Levamisole is a veterinary anthelmintic pharmaceutical, previously used in humans to treat some cancers, and a common adulterant of street cocaine. While it is known to cause agranulocytosis ${ }^{(3)}$, a lesser known public health issue is levamisoleinduced granulomatosis with polyangiitis (previously Wegener's granulomatosis) $)^{(7)}$.

Levamisole-induced GPA, (Wegener's), should be considered in patients with a history of cocaine abuse who present with:

- Unusual extensive destruction of nasal and sinus bony walls with large septal perforation.

- Non-specific symptoms - fever, fatigue, weight loss, night sweats.

- Upper respiratory tract signs - sinus pain, oral and nasal ulcers, nasal septal perforation.

- Microscopic haematuria, oedema and hypertension (due to renal impairment - a very late sign). 


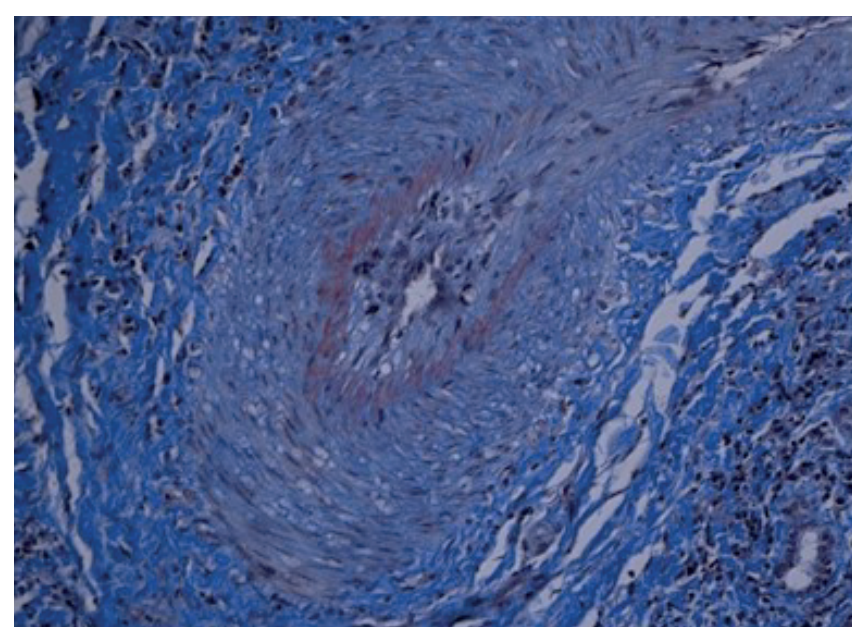

Figure 3. MSB stain. X200 magnification. Showing fibrin deposits and arterial inflammation.

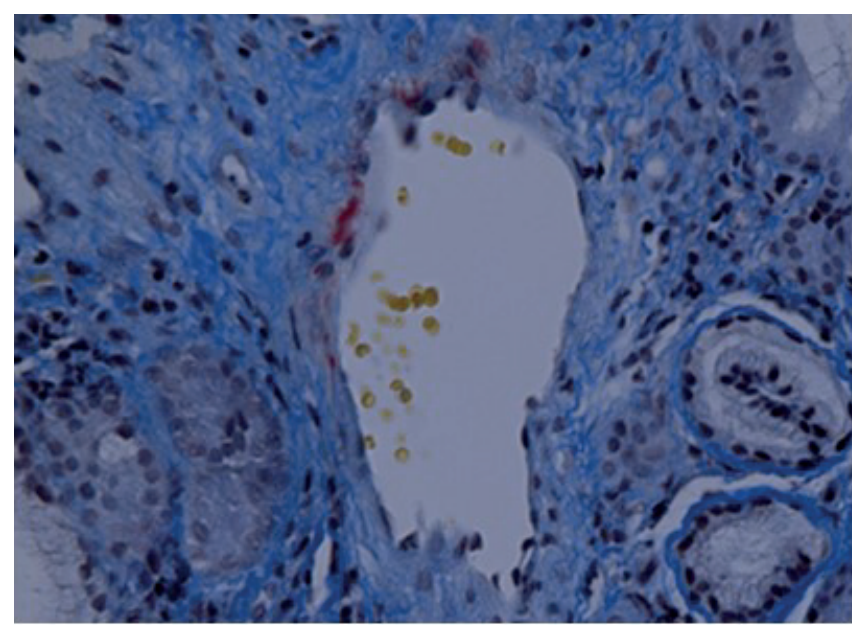

Figure 4. Showing fibrinoid necrosis and vein involvement.

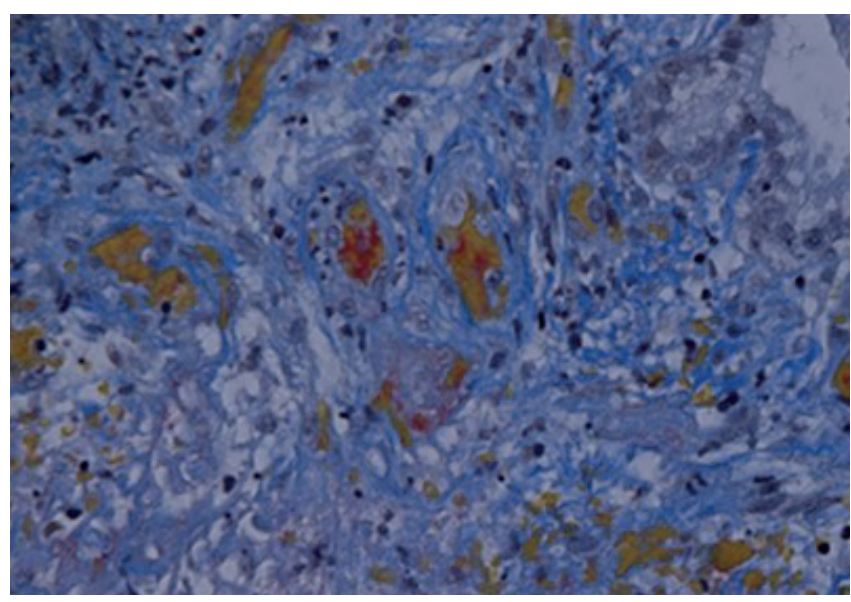

Figure 5. Showing fibirinoid necrosis with small vessel involvement.

- Positive c-ANCA.

These patients should also be checked for neutropenia and levamisole-induced agranulocytosis. Unlike levamisole-induced agranulocytosis, levamisole-induced GPA will not improve following cessation of the use of contaminated cocaine. Without treatment GPA can progress and, as mentioned, lead to significant renal impairment and nasal deformity/destruction ${ }^{(8)}$. It is important that primary and secondary care physicians are aware of this secondary vasculitis, and early diagnosis and aggressive treatment is required.

Our case series highlights a possible link between isolated fibrinoid necrosis of the whole nasal cavity, associated with possible polyangiitis-like changes, and the common cocaine contaminant Levamisole. The presentation, investigation and subsequent management of these patients are similar to that of any suspected vasculitis. This includes good history taking, nasendoscopy, imaging, biopsy and immunological blood sampling. The use of urine toxicology helps to identify the presence of levamisole in the body. These patients benefit from surgical debridement of the affected areas, with quiescence of their ENT manifestations noted on follow-up review. The benefit of systemic treatment with agents such as steroid or methotrexate, is an area which we would like to investigate further along with our rheumatology colleagues. The strongly positive ANCA, along with positive PR3 titres validates the use of systemic treatment for ANCA associated vasculitis, whereas the variable, weaker and less specific ANA and $p$-ANCA positive titres were less reliable

(9). This immunological variability is also seen in cocaine induced septal necrosis.

The list of differential diagnosis for such a presentation includes cocaine use, Wegener's granulomatosis, sarcoidosis and malignancy. Differentiating between these entities relies on history taking, including enquiry into common systemic manifestations of these disease processes, nasendoscopy and biopsy sampling. A differentiating factor between regular pure cocaine-induced necrosis, would be the extent of the nasal cavity destruction; with cocaine necrosis normally confined to nasal septal destruction ${ }^{(10)}$. Conversely, the described levamisole-induced necrosis is more widespread, characterised by its extension posteriorly and into the lateral walls of the nasal cavity.

\section{Conclusion}

Our case series highlights a possible link between levamisole and extensive necrosis of the nasal cavity caused by polyangiitis with granuloma formation and secondary vasculitis. A high degree of suspicion is needed if young patients present with ANCA positive vasculitis or in patients with cocaine use if there is a disproportionate destruction of tissues particularly the lateral wall of the nose. If diagnosed and treated early this can be lifesaving. Although limited to a small series, it is worth considering this diagnosis for extensive lateral wall destruction in addition to septal destruction, in cocaine users. The variability in the vasculitic processes and immunological changes is one that would require 
further investigation, with treatments varying accordingly. These patients benefit from surgical debridement, cessation of cocaine use with or without systemic treatment of vasculitis.

\section{Acknowledgement}

Dr Lucy McGeoch - Rheumatology Consultant who advised on vasculitic results/interpretation/treatment.

\section{Authorship contribution}

DGA: Primary writer of the case series and information collection; RAS: Secondary writer and initial imaging/information collection; ATK: Pathology reports for case series and proof reader NB: ENT Consultant, patient information, report and imaging collection and over-saw report.

\section{Conflict of interest}

There are no conflicts of interest.
1. Chang A, Osterloh J, Thomas J. Levamisole: A Dangerous New Cocaine Adulterant. Clin Pharmacol Therap. 2010; 88 (3): 408-411.

2. Lee KC, Ladzinski B, Nutan FNU. Systemic complications of levamisole toxicity. J Am Acad Dermatol 2012; 67 (4): 791-792.

3. Thompson JS, Herbick JM, Klassen LW et al. Studies on levamisole-induced agranulocytosis. Blood 1980; 56 (3): 388-389.

4. Prokopakis E, Nikolaou V, Vardouniotis A, Jorissen M. Nasal manifestations of systemic diseases. B-ENT. 2013;9(3):171-184.

5. Lazareth H, Peytavin G, Polivka L, Dupin N. The hairy-print for levamisole-induced vasculitis.BMJ Case Rep. 2012 Aug 8;2012. pii: bcr2012006602.

6. Del Buono EA, Flint A. Diagnostic usefulness of nasal biopsy in Wegener's granulomatosis. Hum Pathol 1991; 22(2): 107-110.

7. Kopp SA, High WA, Green JJ. Levamisoleinduced Wegener's granulomatosis following contaminated cocaine abuse. Skin Med 2012; Jul-Aug;10(4):254-256.

8. BMJ Best Practice. Granulomatosis with polyangiitis (Wegener's): Diagnosis. Available from: http://bestpractice.bmj.com/bestpractice/monograph/327/diagnosis.html.

9. Rowshani AT, Schot LJ, ten Berge IJ. c-ANCA as a serological pitfall. Lancet. 2004 Mar 6:363(9411):782.

10. Trimarchi M, Bertazzoni G, Bussi M. Cocaine induced midline destructive lesions. Rhinology. 2014;52(2):104-111.
Natarajan Balaji

ENT Department

Monklands Hospital

Monkscourt Avenue

Airdrie

ML6 0JS Scotland

United Kingdom

Tel: $+44-1236712330$

E-mail: nbalajient@yahoo.com

ISSN: 2589-5613 / @2019 The Author(s). This work is licensed under a Creative Commons Attribution 4.0 International License. The images or other third party material in this article are included in the article's Creative Commons license, unless indicated otherwise in the credit line; if the material is not included under the Creative Commons license, users will need to obtain permission from the license holder to reproduce the material. To view a copy of this license, visit http://creativecommons.org/ licenses/by/4.0/ 